\title{
Albinism and audiogenic seizures in the mouse
}

KEITH GUTHERZ AND D. D. THIESSEN 1

UNIVERSITY OF TEXAS

Albino (cc) and non-albino ( $\mathrm{CC}$ or $\mathrm{Cc}$ ) mice were tested for audiogenic seizure susceptibility beginning at all of the ages from day 14 to day 21 postpartum. At every starting age the onset of first seizure was later for the albino genotype. The latency measures and day of death indicate, however, that the initial retardation is overcome by a faster subsequent development.

Coat color genes have long been suspected to pleiotropically affect behavior in rodents (Keeler \& King, 1942). A strict test of this hypothesis was lacking until Winston \& Lindzey (1964) demonstrated in genetically segregating populations of mice that the recessive allele for albinism on linkage group I retarded water escape performance when in the homozygous state (cc). We are continuing the search for pleiotropic effects of this allele, particularly those that implicate neural functions. This report demonstrates that albino mice, homozygous for cc but unsystematically varying at other loci, are retarded in their development of audiogenic seizure susceptibility.

\section{Method}

Albino (cc) and non-albino ( $\mathrm{Cc}$ or $\mathrm{CC}$ ) mice were culled from $F_{2}$ offspring derived from an $\mathrm{AJ} \times \mathrm{DBA} /{ }_{1}^{\mathrm{J}}$ cross. At 11 days of age standard litters were formed consisting of two albinos and six non-albinos. Litters were randomly assigned so that one litter was tested for seizure proneness beginning on each of the days 14 through 21 postpartum. The exception to this occurred when due to procedural error two litters were tested beginning on day 19. Once an individual was started on a test it was exposed daily to a 60-sec. auditory stimulus from a 5-in. doorbell. The testing chamber consists of a metal waste can, 9 in. in diameter by $11 \mathrm{in.} \mathrm{high,} \mathrm{suspended} \mathrm{by} \mathrm{foam} \mathrm{rubber} \mathrm{in}$ a larger pasteboard drum. A plywood cover, also lined with foam rubber, with an observation window fits over the top of the apparatus. On the inside of the chamber are four 5 in. doorbells and two $5 \mathrm{w}$, $110 \mathrm{v}$ light bulbs. Only one of the bells was used. Litter testing consisted of removing an animal from its cage in the colony room, placing it in the test apparatus in an adjacent room and immediately presenting the auditory stimulus for $60 \mathrm{sec}$. If the animal survived it was placed with its litter mates in a holding cage in the colony room and later returned to the litter condition.

Latencies to first seizure, recovery and death were recorded. The onset of seizure is defined as the beginning of tonus, indicated by the animal falling on its side and kicking or extending its rear legs. Recovery

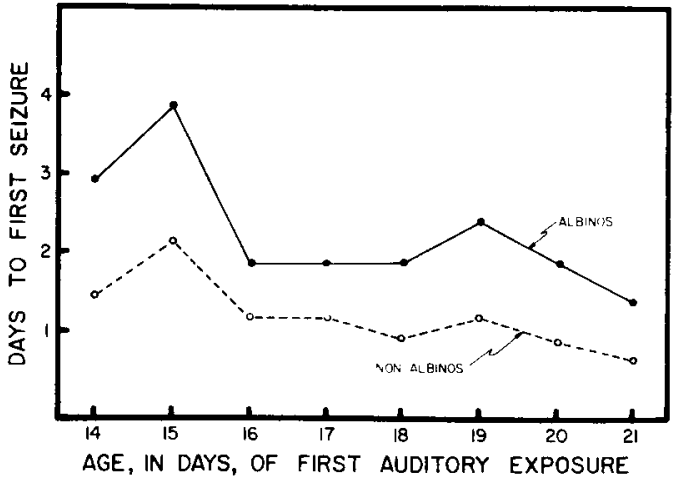

Fig. 1. Number of days to first seizure as a function of age of first test.

is defined as walking on all four legs. Death is inferred from the rising of the flattened pinnae.

Results and Discussion

Regardless of when testing began albinos convulsed later than non-albinos $(F=7.67 ; \mathrm{df}=1 / 14 ; \mathrm{p}<.05)$. As Fig. 1 indicates the two genotypes show a parallel course of development even though test days for the two groups did not necessarily correspond. A similar albino effect appears when age of initial test is ignored and the number of days to first convulsion is recorded. The modal day of first seizure for albino mice is about three and that for the non-albino is about two $\left(X^{2}=16.91\right.$; $\mathrm{df}=1 ; \mathrm{p}<.001$ ).

Although the onset of seizure susceptibility is retarded for the albino animals, their first seizure occurs with a significantly shorter latency and they die on approximately the same day as the non-albino animals. At this time we have only modest confidence in the latency measure because of two deviant points. These findings, however, are consistent with the notion that once the albinos reach seizure threshold they rapidly progress through the developmental cycle already underway in the non-albinos.

The only systematic difference among these animals is at the $\mathrm{C}$ locus. Unless tight linkage is involved the effects on audiogenic seizures can be ascribed to recessive homozygosity at this locus. Other tests involving alternative alleles and a point mutation at this locus on an otherwise homogeneous background (C57BL/6J) will clarify any possibility of linkage. The genetic differences described are more impressive when it is recalled that albino and non-albino mice shared common litter environments. The two other coat 
color variants of this study, browns (CCDD, CcDD, CcDd) and grays (CCdd, Ccdd), did not differ from each other.

At present we offer no explanation for the albino effect. We do, however, regard it as persuasive, developmental in origin and of some possible significance in relating the known biochemistry of pigment formation to neural ontogeny. Interestingly, neither genotype would have been considered seizure-prone at any age if only one exposure had been given. There is an accumulative effect of the auditory stimulus that finally reaches seizure threshold after two or three days. It may be the rate of accumulation that dif- ferentiates the genotypes. Regardless, this study indicates the importance of relatively long-term developmental observations, and the possible research gains derived from isolating major gene effects.

\section{References}

Keeler, C. E., \& King, Helen D. Multiple effects of coat color genes in the Norway rat, with special reference to temperament and domestication. J. comp. Psychol., 1942, 34, 241-250.

Winston, H. D., \& Lindzey, G. Albinism and water escape performance in the mouse. Science, 1964, 144, 189-191.

\section{Note}

1. We wish to acknowledge the helpful suggestions of Gardner Lindzey and the support of NIMH Giant 11030-03. 